\title{
Low PCA3 expression is a marker of poor differentiation in localized prostate tumors: exploratory analysis from 12,076 patients
}

\author{
Mohammed Alshalalfa ${ }^{1}$, Gerald W. Verhaegh ${ }^{2,3}$, Ewan A. Gibb ${ }^{1}$, Maria Santiago- \\ Jiménez ${ }^{1}$, Nicholas Erho ${ }^{1}$, Jennifer Jordan ${ }^{1}$, Kasra Yousefi ${ }^{1}$, Lucia L.C. Lam ${ }^{1}$, Tyler \\ Kolisnik $^{1}$, Jijumon Chelissery ${ }^{1}$, Roland Seiler ${ }^{1,4}$, Ashley E. Ross ${ }^{5}$, R. Jeffrey Karnes ${ }^{6}$, \\ Edward M. Schaeffer ${ }^{7}$, Tamara T. Lotan ${ }^{8}$, Robert B. Den ${ }^{9}$, Stephen J. Freedland ${ }^{10}$, \\ Elai Davicioni ${ }^{1}$, Eric A. Klein ${ }^{11}$ and Jack A. Schalken ${ }^{2,3}$ \\ ${ }^{1}$ GenomeDx Biosciences Inc., Vancouver, BC, Canada \\ ${ }^{2}$ Department of Urology, Radboud University Medical Center, Nijmegen, The Netherlands \\ ${ }^{3}$ Radboud Institute for Molecular Life Sciences, Nijmegen, The Netherlands \\ ${ }^{4}$ Department of Urologic Sciences, University of British Columbia, Vancouver, BC, Canada \\ ${ }^{5}$ James Buchanan Brady Urological Institute, Johns Hopkins Hospital, Baltimore, MD, USA \\ ${ }^{6}$ Department of Urology, Mayo Clinic, Rochester, MN, USA \\ ${ }^{7}$ Department of Urology, Feinberg School of Medicine, Northwestern University, Chicago, IL, USA \\ ${ }^{8}$ Department of Pathology and Oncology, Johns Hopkins School of Medicine, Baltimore, MD, USA \\ ${ }^{9}$ Sidney Kimmel Cancer Centre, Thomas Jefferson University, Philadelphia, PA, USA \\ ${ }^{10}$ Department of Surgery, Division of Urology, Center of Integrated Research on Cancer and Lifestyle, Samuel Oschin \\ Comprehensive Cancer Center, Cedars Sinai Medical Center, Los Angeles, CA, USA \\ ${ }^{11}$ Glickman Urological \& Kidney Institute, Cleveland Clinic, Cleveland, OH, USA \\ Correspondence to: Mohammed Alshalalfa, email: mohamed@genomedx.com \\ Keywords: prostate cancer, PCA3, initial biopsy, prognosis, under-diagnosis \\ Received: November 16, $2016 \quad$ Accepted: January 10, $2017 \quad$ Published: February 07, 2017 \\ Copyright: Alshalalfa et al. This is an open-access article distributed under the terms of the Creative Commons Attribution License \\ 3.0 (CC-BY 3.0), which permits unrestricted use, distribution, and reproduction in any medium, provided the original author and \\ source are credited.
}

\section{ABSTRACT}

Background: Prostate cancer antigen 3 (PCA3) is a prostate cancer diagnostic biomarker that has been clinically validated. The limitations of the diagnostic role of PCA3 in initial biopsy and the prognostic role are not well established. Here, we elucidate the limitations of tissue PCA3 to predict high grade tumors in initial biopsy.

Results: PCA3 has a bimodal distribution in both biopsy and radical prostatectomy (RP) tissues, where low PCA3 expression was significantly associated with high grade disease $(p<0.001)$. PCA3 had a poor performance of predicting high grade disease in initial biopsy (GS $\geq 8$ ) with $55 \%$ sensitivity and high false negative rates; $42 \%$ of high Gleason $(\geq 8)$ samples had low PCA3. In RP, low PCA3 is associated with adverse pathological features, clinical recurrence outcome and greater probability of metastatic progression $(p<0.001)$.

Materials and Methods: A total of 1,694 expression profiles from biopsy and 10,382 from RP patients with high risk tumors were obtained from the Decipher Genomic Resource Information Database (GRIDTM)prostate cancer database. The primary clinical endpoint was distant metastasis-free survival for RP and high Gleason grade for biopsy. Logistic regression analyses and Cox proportional hazards models were used to evaluate the association of PCA3 with clinical variables and risk of metastasis. 


\section{Conclusions: There is high prevalence of high grade tumors with low PCA3 expression in the biopsy setting. Therefore, urologists should be warned that using PCA3 as stand-alone test may lead to high rate of under-diagnosis of high grade disease in initial biopsy setting.}

\section{INTRODUCTION}

Prostate cancer $(\mathrm{PCa})$ accounts for approximately $23 \%$ of all male cancers, and $9 \%$ of all cancer deaths among men in Europe and in Northern America [1]. Only a subset of these patients may harbor aggressive disease with potential recurrence after first-line treatment. To improve patient management, it is essential to use accurate, quantitative tools (i.e genomic biomarkers) to identify patients with aggressive disease. Several recent studies have demonstrated the utility of protein coding and non-coding genes as prognostic and diagnostic biomarkers [2-4]. In particular, a number of long non-coding RNAs (lncRNAs) including Prostate cancer antigen 3 (PCA3), PCATs and SCHLAPI have emerged as attractive diagnostic or prognostic biomarkers $[3,5,6]$.

$P C A 3$ is over-expressed in $95 \%$ of prostate cancers, with up to 100 -fold up-regulation compared to adjacent non-neoplastic cells [7]. PCA3 is also over-expressed in high-grade prostatic intraepithelial neoplasia [8-10]. In clinical practice, urine-based $P C A 3$ testing has been shown to outperform the diagnostic ability of Prostate Specific Antigen (PSA) in men with a prior negative biopsy $[11,12]$ resulting in FDA approval for clinical use for this indication [13]. Urinary PCA3 showed similar performance in initial biopsy setting [14-16]. However, the diagnostic performance of $P C A 3$ in initial biopsy setting has led to high rate of underdiagnosis of high grade disease where $P C A 3$ had a sensitivity of $42 \%$ with a high false negative rate using a cut-off of 60 [16]. In 463 european men scheduled for repeat biopsy, $27(21 \%)$ cancers with a Gleason score of 7-9 would have been missed using a cut-off of 35 [11]. Similarly, $P C A 3$ failed to predict high grade cancers with magnetic resonance imaging (MRI) suspicion score (mSS4-5) in the initial biopsy [17]. These studies illuminate the potential limitations of using urinary $P C A 3$ as a diagnostic biomarker and suggest that some patients with low $P C A 3$ may harbor aggressive disease at initial biopsy.

In the background of the established clinical role of $P C A 3$, the limitations of $P C A 3$ in the initial biopsy setting and the clinical and prognostic utility of tissue $P C A 3$ in radical prostatectomy (RP) setting has not been reported in large cohorts. In this study, we quantify the expression of PCA3 in a large $(\mathrm{n}=12,076)$ localized prostate cancer cohort and relate these data to clinicopathological parameters, generating, to our knowledge, the largest study that correlates $P C A 3$ expression to prostate cancer clinical outcome.
RESULTS

\section{Expression of $P C A 3$ in primary prostate cancer tissue}

Previous studies have evaluated $P C A 3$ in urine [11, 12], yet the characteristics of $P C A 3$ expression in prostate tissue remain under studied [18]. To begin to explore the prostate-specific expression of $P C A 3$, we evaluated publically available The Cancer Genome Atlas (TCGA) data finding that while $P C A 3$ was expressed in very few samples from other cancers, it was most abundant in prostate cancer (Figure 1A).

To expand our initial observations regarding the expression of $P C A 3$ in prostate cancer, we used an arraybased approach to profile a large cohort of 8,532 and 1,694 prostate tumors from prospective RP and biopsy tissues, respectively. For this analysis, we included the wellcharacterized lncRNA SCHLAP1 as control for high grade prostate cancer [3]. As expected, we observed a strong right skewed distribution for SCHLAP1, confirming the reported expression pattern of this gene in prostate cancer (Figure 1B) [3]. In stark contrast, $P C A 3$ demonstrated a bimodal distribution where $23 \%$ and $29 \%$ of patients were categorized as having low PCA3 $(\leq 1.3)$ in biopsy and RP samples respectively (Figure 1B-1C). Gene with bimodal distribution exhibit mainly either high or low expression with small proportion with intermediate expression, unlike normal distribution in which most patients express intermediate expression. This observation was validated using a retrospective cohort of 1,850 patient expression profiles from the Decipher GRID database (Supplementary Figure 2). This bimodality could be a result of tumor cells going through a differentiation process and change of cellular state.

To interpret this observation in the context of prostate cancer genomics, we hypothesized that ERG fusion may be driving the expression of $P C A 3$. However, PCA3 displayed a bimodal distribution in both ERG+ and ERG- tumors, as determined previously by our group [19], suggesting that the observed bimodal distribution is independent of ERG expression (Figure 1D).

\section{High grade tumors in biopsy tissues have low $P C A 3$ expression}

To interpret the bimodal distribution clinically, we first investigated if $P C A 3$ bimodality related to Gleason score in 1,694 initial biopsy samples from the Decipher GRID database. We found $P C A 3$ was significantly associated with Gleason group (Spearman's correlation: $-0.15, \mathrm{p}<0.001$ ) where high Gleason group (Group 4\&5) tumors showing lower expression of $P C A 3$ (Figure 2A). 
Out of 234 samples with Gleason group 4\&5, 105 (44.8\%) had low PCA3 levels which was significantly higher than $18 \%$ (out of 1228) Gleason groups (1\&2) (Fisher's test $\mathrm{p}<0.001)$. Out of 93 samples with Gleason group 5, the percentage of low PCA3 reached 58\%. These results indicate that $P C A 3$ is a poor predictor of high grade disease in biopsy with sensitivity of $55 \%$ and false negative rate of $44.8 \%$ in high grade tumors.

\section{Low $P C A 3$ in RP tissues is associated with adverse pathological variables}

Next, we investigated if low $P C A 3$ relates to clinicopathological variables at RP. In the prospective cohort $(\mathrm{n}=8,532)$ and pooled retrospective cohort $(\mathrm{n}=1,850)$, we found low $P C A 3$ to be associated with high Gleason grades (groups 4\&5) (Spearman's correlation:

A

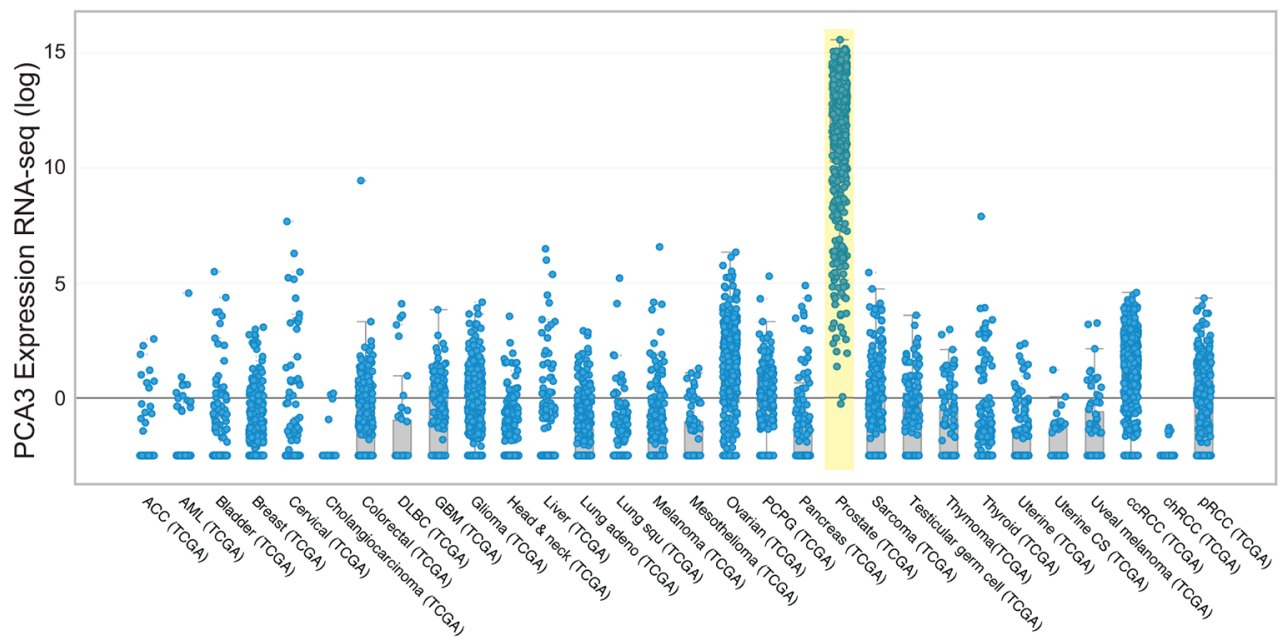

B
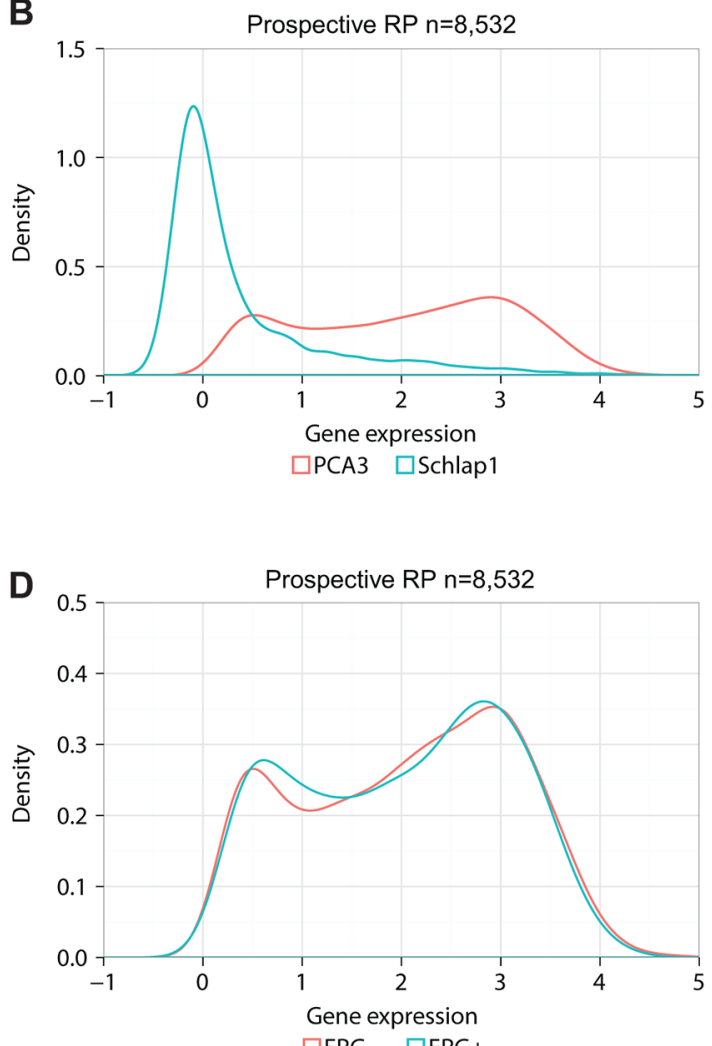

C

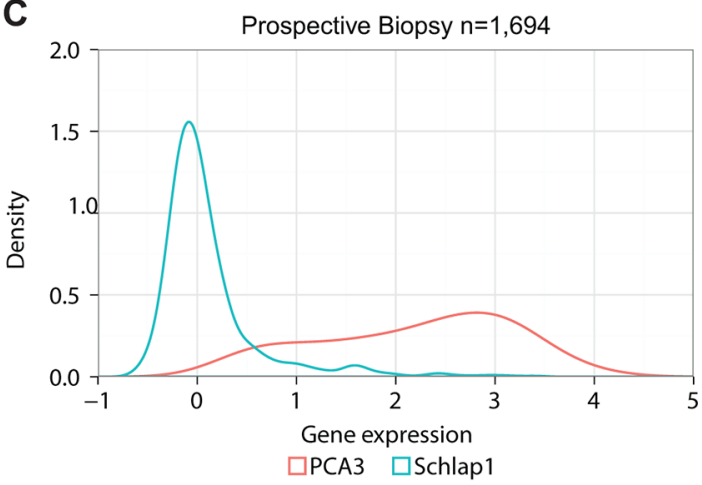

Figure 1: Pan-cancer and prostate-specific expression of $\boldsymbol{P C A 3}$. A. TCGA Pan cancer analysis showing $P C A 3$ to be $P C a$ specific. B. Distribution of $P C A 3$ (red) and SChLAP1 (blue) expression in prospective RP (B) and in biopsy $\mathbf{C}$. showing that $P C A 3$ has a bimodal distribution unlike SChLAP1 that is right skewed. D. Distribution of PCA3 in ERG+ and ERG- tumors as determined by our group previously [19] in prospective RP cohort. 
$-0.18, \mathrm{p}<0.001$ ) (Figure 2B). In the prospective cohort, out of 1,790 patients in the Gleason groups 4\&5, 763 (42\%) have low $P C A 3$. These results confirmed high levels of $P C A 3$ was a poor predictor of high grade disease and low $P C A 3$ levels was not an indication of the absence of cancer.

Distribution plots of $P C A 3$ across Gleason groups showed Gleason groups 1 and 2 had a right modal distribution for $P C A 3$, while group 5 (GS 9-10) had left modal distribution, in both the prospective biopsy (Figure 2C), prospective RP (Figure 2D) and retrospective RP cohorts (Supplementary Figure 3). The distributions for GS $4+3$ and GS 8 were bimodal, however, the bimodal distribution for GS $3+4$ was skewed to the right (Figure 2C). These data suggest the expression of $P C A 3$ expression in prostate tissue may reflect the differentiation status of tumors, correlated with the amount of pattern 4 tumor present in tumors with mixed Gleason grade.

Next, we explored the association of $P C A 3$ expression (low vs. high) with pathological tumor characteristics, including pathological stage and Gleason group (Table 1). In the prospective, Gleason group 5 (Odd Ratio (OR): 3.88, 95\% Confidence Interval (CI): 3.09-4.86, $\mathrm{p}<0.001)$ when compared to group 1, Seminal Vesicle Invasion (SVI) (OR: 2.12 [1.89-2.39], $\mathrm{p}<0.001$ ), Extra Prostatic Extension (EPE) (OR: 1.59 [1.44-1.75], $\mathrm{p}<0.001$ ) and Lymph Node Invasion (LNI) (OR: 2.14 [1.72-2.66], $\mathrm{p}<0.001)$ were significantly associated with low PCA3 on Univariable Analysis (UVA). Similar results were obtained using the retrospective cohort (Table 1). Using Multivariable Analysis (MVA) logistic regression, EPE, SVI and Gleason group 5 remained significantly associated with $P C A 3$ expression in the prospective and retrospective cohorts (Table 1). The negative correlation between $P C A 3$ expression and Gleason scores was also observed in urinary samples $(\mathrm{n}=50)$ (Supplementary Figure 4) where $P C A 3$ expression was measured using the same Human Exon arrays used for PCA3 expression measurement in RP and biopsy tissues.
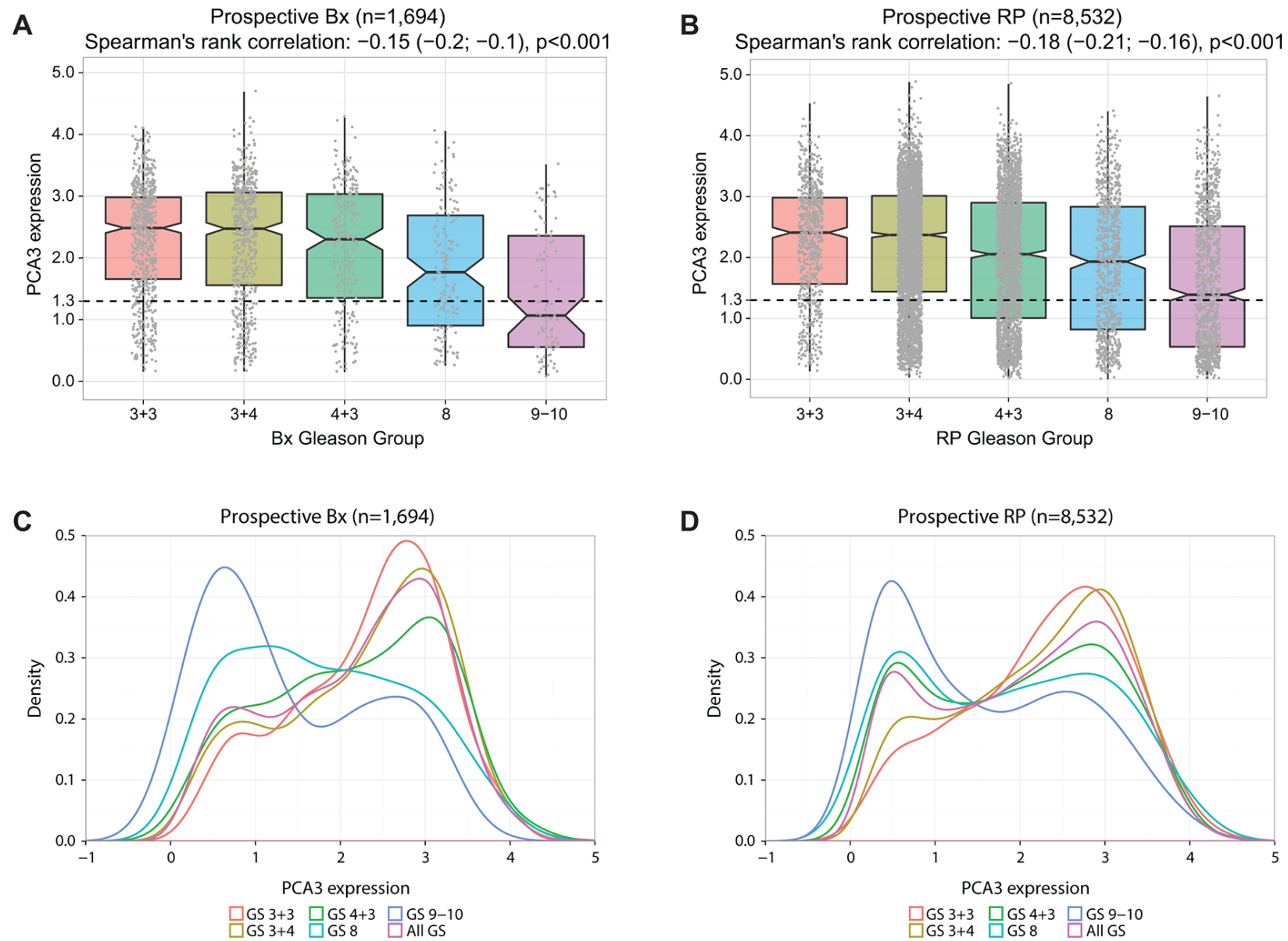

Figure 2: Associations of PCA3 expression and pathological characteristics at RP. A. $P C A 3$ expression across Gleason groups $(1-5)$ in biopsy samples $(n=1,694)$ and B. RP samples $(n=8,532)$ showing that around $50 \%$ of Gleason 5 group have low $P C A 3$. C-D. Distribution of $P C A 3$ across Gleason groups in biopsy (C) and RP samples (D) showing GS5 to be more enriched with low $P C A 3$ while GS1 enriched more with high $P C A 3$. 
Table 1: Univariable and multivariable analysis associating clinicopathologic risk factors with PCA3 in prospective $(n=8,532)$ and retrospective cohorts $(n=1,850)$

\begin{tabular}{|c|c|c|c|c|}
\hline & \multicolumn{4}{|c|}{ Prospective data $(n=8,532)$} \\
\hline & \multicolumn{2}{|l|}{ UVA } & \multicolumn{2}{|l|}{ MVA } \\
\hline & Estimate(95\% CI) & p value & Estimate $(95 \% \mathrm{CI})$ & p value \\
\hline pre-PSA (ref: $<10$ ng/ml) & $1.21(1.05-1.4)$ & 0.008 & $0.91(0.78-1.07)$ & 0.26 \\
\hline $\mathrm{EPE}$ & $1.59(1.44-1.75)$ & $<0.001$ & $1.24(1.07-1.43)$ & 0.005 \\
\hline SVI & $2.12(1.89-2.39)$ & $<0.001$ & $1.61(1.35-1.92)$ & $<0.001$ \\
\hline SM & $0.98(0.89-1.08)$ & 0.73 & $0.97(0.85-1.12)$ & 0.69 \\
\hline LNI & $2.14(1.72-2.66)$ & $<0.001$ & $1.42(1.05-1.91)$ & 0.02 \\
\hline $\begin{array}{l}\text { Gleason group } 2 \text { (ref: } \\
\text { group 1) }\end{array}$ & $1.21(0.99-1.49)$ & 0.07 & $1.1(0.77-1.57)$ & 0.6 \\
\hline $\begin{array}{l}\text { Gleason group } 3 \text { (ref: } \\
\text { group 1) }\end{array}$ & $1.96(1.59-2.41)$ & $<0.001$ & $1.58(1.1-2.27)$ & 0.01 \\
\hline $\begin{array}{l}\text { Gleason group } 4 \text { (ref: } \\
\text { group 1) }\end{array}$ & $2.33(1.83-2.96)$ & $<0.001$ & $1.83(1.23-2.72)$ & 0.003 \\
\hline \multirow[t]{4}{*}{$\begin{array}{l}\text { Gleason group } 5 \text { (ref: } \\
\text { group 1) }\end{array}$} & $3.88(3.09-4.86)$ & $<0.001$ & $2.83(1.93-4.15)$ & $<0.001$ \\
\hline & \multicolumn{4}{|c|}{ Pooled retrospective data $(n=1,850)$} \\
\hline & \multicolumn{2}{|c|}{ UVA } & \multicolumn{2}{|c|}{ MVA } \\
\hline & Estimate (95\% CI) & p value & Estimate (95\% CI) & p value \\
\hline pre-PSA (ref: <10 ng/ml) & $0.9359(0.756-1.158)$ & 0.542 & $0.8036(0.638-1.012)$ & 0.064 \\
\hline EPE & $1.3662(1.112-1.679)$ & 0.003 & $1.1358(0.901-1.433)$ & 0.282 \\
\hline SVI & $1.6435(1.312-2.059)$ & $<0.001$ & $1.3382(1.031-1.737)$ & 0.028 \\
\hline $\mathrm{SM}$ & $0.8033(0.659-0.979)$ & 0.030 & $0.7931(0.640-0.983)$ & 0.035 \\
\hline LNI & $1.8508(1.353-2.532)$ & $<0.001$ & $1.2657(0.887-1.807)$ & 0.194 \\
\hline $\begin{array}{l}\text { Gleason group } 2 \& 3 \text { (ref: } \\
\text { group 1) }\end{array}$ & $0.9361(0.699-1.253)$ & 0.657 & $0.9123(0.651-1.278)$ & 0.593 \\
\hline $\begin{array}{l}\text { Gleason group } 4 \text { (ref: } \\
\text { group 1) }\end{array}$ & $1.8648(1.272-2.734)$ & 0.001 & $1.7886(1.177-2.718)$ & 0.006 \\
\hline $\begin{array}{l}\text { Gleason group } 5 \text { (ref: } \\
\text { group 1) }\end{array}$ & $2.1513(1.542-3.001)$ & $<0.001$ & $1.951(1.320-2.883)$ & $<0.001$ \\
\hline
\end{tabular}

pre-PSA: preoperative prostate specific antigen; EPE: extra prostatic extension; SVI: seminal vesicle invasion; SM: surgical margins; LNI: lymph node invasion.

\section{Low $P C A 3$ expression tracks with poor prognosis and metastasis after RP}

To assess the association of $P C A 3$ expression with clinical endpoints we plotted $P C A 3$ expression in a John Hopkins Medical Institute (JHMI) cohort previously used to validate the Decipher test (JHMI-I) [20] and tagged events for biochemical recurrence (BCR), metastasis (MET) and Prostate Cancer Specific Mortality (PCSM) free survival (Figure 3A). Here, we observed patients with low $P C A 3$ expression had lower 5-year BCR free survival rates (58\%), 5-year MET free survival rates $(75 \%)$ and 10 year PCSM free survival $(85 \%)$ compared to high $P C A 3$ patients in JHMI-I cohort $(76,92,96 \%$ for BCR, MET and PCSM respectively) (Figure 3A, Supplementary Table 3). A similar trend was observed in the Mayo-Clinic II cohort that was used to validate Decipher test (Supplementary Table 3).

In a set of 665 patients pooled from two cohorts $[21,22]$ and the subcohorts of two previously published 
case cohorts [20, 23], we found patients with high PCA3 had nearly $50 \%$ lower risk of developing metastasis at 5-years, compared to those with low PCA3 (Hazard Ratio (HR): 0.56 (95\% CI: 0.43-0.72, p<0.001) (Figure 3B). Kaplan-Meier analysis showed that low PCA3 is significantly associated with early metastasis after radical prostatectomy in both the JHMI-I (HR: 0.35, 95\% CI:
0.22-0.57, $\mathrm{p}<0.001)$ and Mayo-Clinic II (HR: 0.45, 95\% CI: $0.26-0.79, p=0.005$ ) datasets (Figure 3C, 3D). In the prospective RP cohort outcome data are currently not available, making it difficult to assess the prognostic impact of $P C A 3$. However, in previous work we have demonstrated a robust correlation between Decipher risk categories and metastasis [20]. As such, we used the
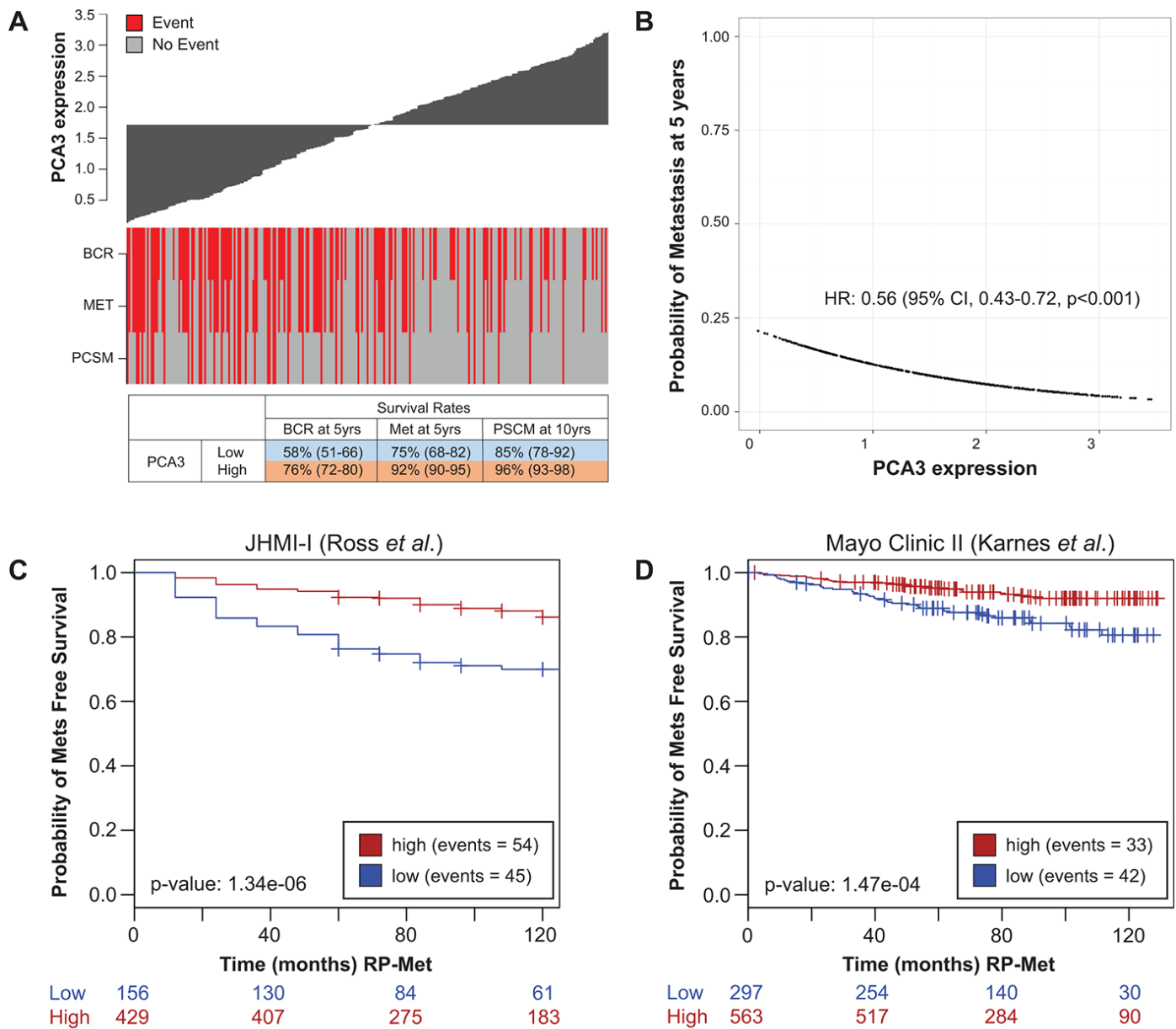

E

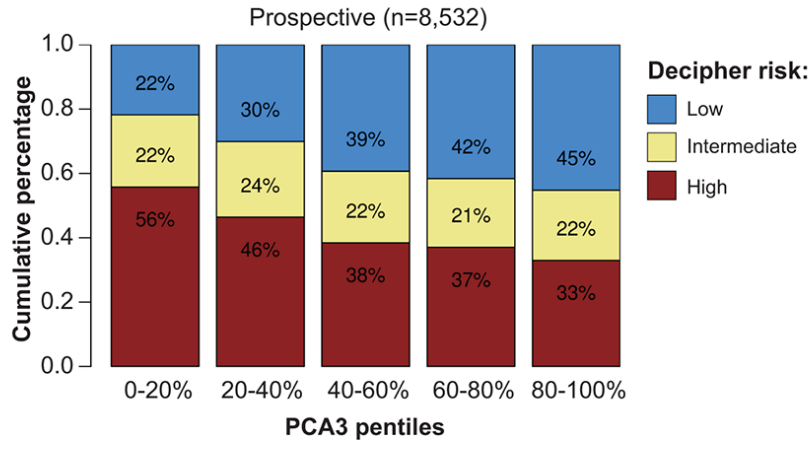

Figure 3: Prognostic impact of $\boldsymbol{P C A 3}$ in localized prostate tumors. A. Waterfall plot of $P C A 3$ with clinical endpoint annotations. BCR, biochemical recurrence; MET, metastasis, PCSM, PCa-specific mortality. B. Association between probability of 5-year metastasis and PCA3 expression in 665 samples pooled from two cohorts [21, 22] and the subcohorts from two case cohorts (JHMI-I, Mayo-Clinic II) [20, 23]. C-D. Kaplan Meier analysis in two case-cohorts from JHMI-I and Mayo-Clinic II. PCA3 expression was categorized into low vs high. E. Association between Decipher risk categories and $P C A 3$ levels showing low $P C A 3$ patients are more enriched with high risk Decipher. 
frequency of Decipher risk categories as a surrogate for metastasis (low, intermediate vs high risk) across $P C A 3$ expression pentiles (5 categories of $P C A 3$ expression each with $20 \%$ of samples). Using these metrics, we observed $56 \%$ and $33 \%$ of patients with high Decipher showed low and high $P C A 3$ expression, respectively (Fisher's test, $\mathrm{p}<0.001$ ) (Figure 3E). Interestingly, one of the top positively correlated genes across 8,532 samples is PCAT-14 $(\mathrm{R}=0.32, \mathrm{p}<0.0001)$, which has also a bimodal distribution (Supplementary Figure 5) and has shown to increase migration [5]. Additional analysis found genes negatively correlated to $P C A 3$ expression were enriched in gene sets related to epithelial-to-mesenchymal transition (EMT) based on Gene Set Enrichment Analysis (Supplementary Figure 6). Collectively, these data provide supporting evidence indicating lower $P C A 3$ levels correlate with a higher risk of metastasis and more aggressive prostate cancer at RP and biopsy setting.

\section{DISCUSSION}

The clinical utility of the $P C A 3$ has been established as an independent diagnostic biomarker to predict prostate cancer in initial and repeat biopsy setting, primarily through urine-based analyses [6, 14]. However, the performance of $P C A 3$ in initial biopsy setting has been shown in several studies to be poor with high false negative rate and low sensitivity rate leading to underdiagnosis of high grade disease [11, 16, 24]. Additionally, establishing the prognostic significance of the same assay has been challenging, in part due to small cohort sizes.

In this study, we utilize the large collection of biopsy and RP expression profiles from the Decipher GRID database (GenomeDx Biosciences Inc.) to study PCA3 expression and link it to clinical data and differentiation levels of tumor. We found that $P C A 3$ demonstrated a striking bimodal distribution in prostate tissues representing distinct differentiation levels, which was not associated with ERG status and is distinct from other prostate specific prognostic lncRNA (i.e SChLAP1) that is right skewed. These data suggest $P C A 3$ expression may be indicating two distinct differentiation cellular or tissue states within the prostate tumor; an early state where high levels of $P C A 3$ are detectable and a second, late stage state where $P C A 3$ levels decline. The bimodal expression pattern suggests unique biology for $P C A 3$ which is not consistent with a ramping up of expression, as was observed for SCHLAP1. This observation motivated us to further understand the biology and clinical implication of $P C A 3$ cycle.

We have associated $P C A 3$ with Gleason scores in large biopsy and RP cohorts from the Decipher GRID and found patients with high Gleason have lower PCA3 expression (42-45\%). These results indicate that most patients with high Gleason would have been reported negative on diagnostic biopsy if $P C A 3$ biomarkers was used for initial diagnosis. Moreover, in the prospective cohort from Decipher GRID (RP, biopsy) where metastatic outcome was not available, we found that low $P C A 3$ was strongly associated with high risk Decipher scores and was a surrogate for metastasis. Additional analyses showed that genes negatively correlated with $P C A 3$ are associated with migration pathways such as EMT. Based on expression correlation, PCAT-14 was the top correlated genes. In a recent report [5], In-vitro analysis showed that low PCAT14 expression increased migration while overexpressing PCAT-14 reduced cellular growth, migration, and invasion. These results suggest that patients with low $P C A 3$ are at a significantly higher risk of developing metastasis after RP.

Recent work $[25,26]$ proposed that targeting $P C A 3$ may be a putative therapeutic option to inhibit $\mathrm{PCa}$ growth based on interfering siRNA in LNCaP and PC3 cells. These results are contradicting our observation possibly due the different nature of genomics of LNCaP cells and RP/biopsy tissues (used in this cohort) and different AR-activity in these specimens. We observed strong positive correlation between $P C A 3$ and $A B C C 4, K L K 2$ and $K L K 3$ that are AR target genes supporting the modulatory effect between $A R$ and $P C A 3$. Activated androgen receptor (AR) also strongly induces $P C A 3$ transcription $[26,27]$. We believe that the interaction between $A R$-axis induced by testosterone and $P C A 3$ may significantly elucidate the functional impact of $P C A 3$. Low pre-treatment free testosterone levels, are significantly associated with tumor grade and stage, with lower testosterone levels in patients with high grade $\mathrm{PCa}$ [27]. Prostate cancer patients with lower circulating free testosterone levels also have poor prognosis factors and higher tumor burden prior to treatment [28]. These findings reinforce the idea that low $P C A 3$ levels in high grade $\mathrm{PCa}$ may reflect lower circulating androgen levels, leading to weaker $A R$ activation and transcriptional activity. This suggests that $P C A 3$ may act as a surrogate marker for serum testosterone.

Based on results presented in our study, $P C A 3$ assays as currently formatted have limited utility in detecting men with higher grade disease. Even though such tools have shown to reduce number of biopsies, they have high rate of missing high grade disease due to low PCA3 levels. We have observed such a case recently, where a patient was negative for prostate cancer as assessed by urinary $P C A 3$, but was later diagnosed to have very high grade disease (GS 9) and high Decipher metastasis risk. To overcome this limitation, recently developed commercial diagnostic tools have incorporated additional biomarkers like ERG [7], HOXC6, DLX1 [28]. Additional markers designed to detect higher grade disease and non-ERG tumors would likely improve the utility of the assay. This study suggests that next-generation diagnostic tools using PCA3 and ERG should incorporate additional biomarkers to ensure high grade disease is detected in low PCA3 and ERG-fusion negative samples.

The current study has some limitations. We acknowledge the majority of our analyses are based on tissue PCA3 whilst current PCA3 assays are urine based. We have generated preliminary data profiling gene 
expression from a small number of urine samples $(n=50)$ using the same Human Exon arrays (Supplementary Figure 4). Even though the range of $P C A 3$ expression is different in tumor tissue and urine samples, the $P C A 3$ trend in urine sample is very similar to tissue $P C A 3$ suggesting that results from this study may hold true when conducted in large number of urine sample. Additional studies are warranted to firmly establish the relationship between high grade prostate cancer and the utility of $P C A 3$ score in urine-based diagnostic tests. Additional urine-based diagnostic tools that incorporates additional biomarkers are needed to overcome limitations of $P C A 3$ assays.

\section{MATERIALS AND METHODS}

\section{Study population}

A total of 10,382 expression profiles from RP tissues, primarily with adverse pathology and other high risk features, and 1,694 profiles from initial biopsy tissues were obtained from the Decipher GRID ${ }^{\mathrm{TM}}$ prostate cancer database (NCT02609269). This cohort represents cases from multi-institutional retrospective cohorts (Supplementary Table 1) [2, 20, 21, 23, 29] with detailed clinical, pathological, treatment and outcomes data and de-identified, anonymized prospective cases with basic demographic and pathological data from clinical use of the Decipher test from RP and biopsy settings (Supplementary Table 2).

\section{Tissue processing, microarray data preprocessing and PCA3 expression measurement}

Specimen selection, RNA extraction, and Human Exon 1.0 ST Array hybridization was done in a Clinical Laboratory Improvement Amendments (CLIA/CAP)certified laboratory facility (GenomeDx Biosciences, San Diego, CA, USA) as previously described [30]. The Supplementary Material provides additional details. PCA3 gene expression was summarized using the mean of the 5 probe sets in the Human Exon 1.0 ST array that are falling in the PCA3 locus (Supplementary Figure 1) and corrected for batch effects using an empirical Bayes framework (ComBat in "sva" R package).

\section{Statistical analysis}

Statistical analyses were performed in R v3.2.2, and all tests were two-sided using a 5\% significance level. Clinical variables were categorized as follows: Gleason Score (GS) was grouped into ISUP groups: Group 1(GS 6), Group 2(3+4), Group 3(4+3), Group 4(8), Group 5 (9-10). Pre-operative PSA was categorized into low $(\leq 10 \mathrm{ng} / \mathrm{ml})$ and high $(>10 \mathrm{ng} / \mathrm{ml})$ groups. The following variables were binary (present vs. absent): Extra-prostatic extension (EPE), seminal vesicle invasion (SVI), surgical margins (SM) and lymph node invasion (LNI). Fisher's exact test was used to study the association between categorical variables. $P C A 3$ expression was dichotomized using the optimal cut-off value splitting $P C A 3$ into low $(\leq 1.3)$ vs high $(>1.3)$ using the "optimize R" package that is based on combination of golden section search and successive parabolic interpolation. Correlations of $P C A 3$ expression with pathologic stage and pathologic Gleason score were computed using Spearman's rank correlation. Univariable (UVA) and multivariable (MVA) logistic regression analyses were used to evaluate the association of $P C A 3$ and clinical variables. Kaplan Meier curves stratified by $P C A 3$ expression levels (low vs. high) were constructed to obtain metastasis-free survival rates after RP. Cox proportional hazards analysis were used to determine the performance of $P C A 3$ in predicting risk of metastasis. In time to event analyses, event times were defined as the time from RP to metastasis. Decipher risk categories, an extensively validated measure of prostate cancer metastasis risk [20, 22, 23, 29-32], were used as a surrogate for metastasis (low, intermediate vs high risk) in the prospective cohort where no metastasis outcomes were available.

\section{CONCLUSION}

In conclusion, we report that high levels of $P C A 3$ may be a poor predictor of high grade disease in the initial biopsy setting and low $P C A 3$ expression in primary tumors may portend a poor prognosis. We observed a unique bimodal distribution for $P C A 3$, where low levels of $P C A 3$ were associated with high grade disease in urine, tissue biopsy, and tissue RP, and poor survival and increased rates of metastasis after RP. To our knowledge, this is the largest study characterizing the associations between PCA3 RNA from RP tissues with pathological variables at RP and clinical outcome. Results in this study suggest that sole use of $P C A 3$ as a stand-alone marker for prostate cancer may provide false negatives for patients with higher grade disease and urologists should be warned of this limitation.

\section{Abbreviations}

PCA3: prostate cancer antigen 3; RP: radical prostatectomy; PSA: prostate specific antigen; GRID: genomic resource information database; MVA: multivariable analysis; UVA: univariable analysis; EPE: extra prostatic extension; LNI: lymph node invasion; SVI: seminal vesicle invasion; BCR: biochemical recurrence; MET: metastasis; PCSM: prostate cancer specific mortality. 


\section{Author contributions}

MA, GV, EG, MS, NE, JJ, KY, ED, JS, EK drafted the manuscript, conceived and coordinated the study, and were responsible for interpretation of data. AR, TL, RD, SF, EK, ES, and JK provided patient material and data. LL, TK, JC, RC collected and preprocessed data. All authors read and approved the final manuscript.

\section{ACKNOWLEDGMENTS}

GenomeDX funded specimen collection, performed the Decipher assay, participated in study and analysis design, and participated in manuscript revision, revision and the decision to submit for publication.

\section{CONFLICTS OF INTEREST}

The authors declare that there is no conflicts of interest regarding the publication of this paper.

\section{FINANCIAL DISCLOSURE}

M.A, E.A.G., N.E., J.J, K.Y, M.S, E.D are employees of GenomeDx. J.A.S. and G.W.V. are inventors on PCA3 related IP. The IP is owned by their employer, Radboudumc, who has licensed the technology and receives royalty payments.

\section{REFERENCES}

1. Torre LA, Bray F, Siegel RL, Ferlay J, Lortet-tieulent J, Jemal A. Global cancer statistics, 2012. CA Cancer J Clin. 2015; 65:87-108. doi:10.3322/caac.21262.

2. Erho N, Crisan A, Vergara IA, Mitra AP, Ghadessi M, Buerki C, Bergstralh EJ, Kollmeyer T, Fink S, Haddad Z, Zimmermann B, Sierocinski T, Ballman KV, et al. Discovery and validation of a prostate cancer genomic classifier that predicts early metastasis following radical prostatectomy. PLoS One. 2013; 8:e66855. doi:10.1371/ journal.pone.0066855.

3. Prensner JR, Zhao S, Erho N, Schipper M, Iyer MK, Dhanasekaran SM, Magi-Galluzzi C, Mehra R, Sahu A, Siddiqui J, Davicioni E, Den RB, Dicker AP, et al. RNA biomarkers associated with metastatic progression in prostate cancer: a multi-institutional high-throughput analysis of SChLAP1. Lancet Oncol. 2014; 15:1469-80. doi:10.1016/S1470-2045(14)71113-1.

4. Prensner JR, Iyer MK, Sahu A, Asangani IA, Cao Q, Patel L, Vergara IA, Davicioni E, Erho N, Ghadessi M, Jenkins RB, Triche TJ, Malik R, et al. The long noncoding RNA SChLAP1 promotes aggressive prostate cancer and antagonizes the SWI/SNF complex. Nat Genet. 2013; 45:1392-8. doi:10.1038/ng.2771.
5. White N, Zhao S, Zhang J, Rozycki E, Dang H, McFadden S, Eteleeb AM, Alshalalfa M, Vergara IA, Erho N, Arbeit JM, Karnes RJ, Den RB, et al. Multi-institutional analysis shows that low PCAT-14 expression associates with poor outcomes in prostate cancer. Eur Urol. 2017; 71:257-66.

6. Loeb S, Partin AW. Review of the literature: PCA3 for prostate cancer risk assessment and prognostication. Rev Urol. 2011; 13:e191-5.

7. Salagierski M, Schalken JA. PCA3 and TMPRSS2-ERG: promising biomarkers in prostate cancer diagnosis. Cancers (Basel). 2010; 2:1432-40. doi:10.3390/cancers2031432.

8. Bussemakers MJG, Van Bokhoven A, Verhaegh GW, Smit FP, Karthaus HFM, Schalken JA, Debruyne FM, Ru N, Isaacs WB. DD3: a new prostate-specific gene, highly overexpressed in prostate cancer. Cancer Res. 1999; 59:5975-9. doi:10.1038/ncb2161.

9. de Kok JB, Verhaegh GW, Roelofs RW, Hessels D, Kiemeney LA, Aalders TW, Swinkels DW, Schalken JA. DD3PCA3, a very sensitive and specific marker to detect prostate tumors. Cancer Res. 2002; 62:2695-8.

10. Popa I, Fradet Y, Beaudry G, Hovington H, Beaudry G, Têtu B. Identification of PCA3 (DD3) in prostatic carcinoma by in situ hybridization. Mod Pathol. 2007; 20:1121-7. doi:10.1038/modpathol.3800963.

11. Haese A, de la Taille A, van Poppel, H Marberger M, Stenzl A, Mulders P, Huland H, Abbou CC, Remzi M, Tinzl M, Feyerabend S, Stillebroer AB, van Gils MP, Schalken JA. Clinical utility of the PCA3 urine assay in European men scheduled for repeat biopsy. Eur Urol. 2008; 54:1081-8.

12. Marks LS, Fradet Y, Lim Deras I, Blase A, Mathis J, Aubin SM, Cancio AT, Desaulniers M, Ellis WJ, Rittenhouse H, Groskopf J. PCA3 molecular urine assay for prostate cancer in men undergoing repeat biopsy. Urology. 2007; 69:532-5. doi:10.1016/j.urology.2006.12.014.

13. Hessels D, Klein Gunnewiek J, van Oort I, Karthaus H, van Leenders G, van Balken B, Kiemeney LA, Witjes JA, Schalken JA. DD3(PCA3)-based molecular urine analysis for the diagnosis of prostate cancer. Eur Urol. 2003; 44:8-15.

14. Elshafei A, Chevli KK, Moussa AS, Kara O, Chueh SC, Walter P, Hatem A, Gao T, Jones JS, Duff M. PCA3-based nomogram for predicting prostate cancer and high grade cancer on initial transrectal guided biopsy. Prostate. 2015; 75:1951-7. doi:10.1002/pros.23096.

15. Roobol MJ, Schröder FH, van Leenders GL, Hessels D, Van Den Bergh RCN, Wolters T, van Leeuwen PJ. Performance of prostate cancer antigen 3 (PCA3) and prostate-specific antigen in prescreened men: reproducibility and detection characteristics for prostate cancer patients with high PCA3 scores ( $\geq 100$ ). Eur Urol. 2010; 58:893-9. doi:10.1016/j. eururo.2010.09.030.

16. Wei JT, Feng Z, Partin AW, Brown E, Thompson I, Sokoll L, Chan DW, Lotan Y, Kibel AS, Busby JE, Bidair M, Lin DW, Taneja SS. Can urinary PCA3 Supplement PSA in 
the early detection of prostate cancer? J Clin Oncol. 2014; 32:4066-72. doi:10.1200/JCO.2013.52.8505.

17. Fenstermaker M, Mendhiratta N, Bjurlin MA, Meng X, Rosenkrantz AB, Huang R, Deng FM, Zhou M, Huang WC, Lepor H, Taneja SS. Risk stratification by urinary PCA3 testing prior to MRI-US fusion-targeted prostate biopsy among men with no previous history of biopsy. Urology. 2017; 99:174-9.

18. Warrick JI, Tomlins SA, Carskadon SL, Young AM, Siddiqui J, Wei JT, Chinnaiyan AM, Kunju LP, Palanisamy N. Evaluation of tissue PCA3 expression in prostate cancer by RNA in situ hybridization-a correlative study with urine PCA3 and TMPRSS2-ERG. Mod Pathol. 2014; 27:609-20. doi:10.1038/modpathol.2013.169.

19. Tomlins SA, Alshalalfa M, Davicioni E, Erho N, Yousefi K, Zhao S, Haddad Z, Den RB, Dicker AP, Trock BJ, DeMarzo AM, Ross AE, Schaeffer EM, et al. Characterization of 1577 primary prostate cancers reveals novel biological and clinicopathologic insights into molecular subtypes. Eur Urol. 2015; 68:555-67. doi:10.1016/j.eururo.2015.04.033.

20. Ross AE, Johnson MH, Yousefi K, Davicioni E, Netto GJ, Marchionni L, Fedor HL, Glavaris S, Choeurng V, Buerki C, Erho N, Lam LL, Humphreys EB, et al. Tissue-based genomics augments post-prostatectomy risk stratification in a natural history cohort of intermediate- and high-risk men. Eur Urol. 2016; 69:157-65. doi:10.1016/j.eururo.2015.

21. Den RB, Feng FY, Showalter TN, Mishra MV, Trabulsi EJ, Lallas CD, Gomella LG, Kelly WK, Birbe RC, McCue PA, Ghadessi M, Yousefi K, Davicioni E, et al. Genomic prostate cancer classifier predicts biochemical failure and metastases in patients after postoperative radiation therapy. Int J Radiat Oncol Biol Phys. 2014; 89:1038-46. doi:10.1016/j.ijrobp.2014.04.052.

22. Klein EA, Yousefi K, Haddad Z, Choeurng V, Buerki C, Stephenson AJ, Li J, Kattan MW, Magi-Galluzzi C, Davicioni E. A genomic classifier improves prediction of metastatic disease within 5 years after surgery in nodenegative high-risk prostate cancer patients managed by radical prostatectomy without adjuvant therapy. Eur Urol. 2015; 67:778-86. doi:10.1016/j.eururo.2014.10.036.

23. Karnes RJ, Bergstralh EJ, Davicioni E, Ghadessi M, Buerki C, Mitra AP, Crisan A, Erho N, Vergara IA, Lam LL, Carlson R, Thompson DJ, Haddad Z, et al. Validation of a genomic classifier that predicts metastasis following radical prostatectomy in an at risk Patient population. J Urol. 2013; 190:2047-53. doi:10.1016/j.juro.2013.06.017.

24. Ruffion A, Devonec M, Champetier D, Decaussin-Petrucci M, Rodriguez-Lafrasse C, Paparel P, Perrin P, VlaeminckGuillem V. PCA3 and PCA3-based nomograms improve diagnostic accuracy in patients undergoing first prostate biopsy. Int J Mol Sci. 2013; 14:17767-80. doi:10.3390/ ijms 140917767.

25. Lemos AEG, Ferreira LB, Batoreu NM, de Freitas PP, Bonamino MH, Gimba ERP. PCA3 long noncoding RNA modulates the expression of key cancer-related genes in LNCaP prostate cancer cells. Tumor Biol. 2016; 37:1133948. doi:10.1007/s13277-016-5012-3.

26. Ferreira L, Palumbo A, de Mello K, Sternberg C, Caetano M, de Oliveira F, Neves AF, Nasciutti LE, Goulart LR, Gimba ER. PCA3 noncoding RNA is involved in the control of prostate-cancer cell survival and modulates androgen receptor signaling. BMC Cancer. 2012; 12:507.

27. Salagierski M, Verhaegh GW, Jannink SA, Smit FP, Hessels D, Schalken JA. Differential expression of PCA3 and its overlapping PRUNE2 transcript in prostate cancer. Prostate. 2010; 70:70-8. doi:10.1002/pros.21040.

28. Van Neste L, Hendriks RJ, Dijkstra S, Trooskens G, Cornel EB, Jannink SA, de Jong H, Hessels D, Smit FP, Melchers WJ, Leyten GH, de Reijke TM, Vergunst H, et al. Detection of high-grade prostate cancer using a urinary molecular biomarker-based risk score. Eur Urol. 2016; 70:740-48. doi:10.1016/j.eururo.2016.04.012.

29. Freedland SJ, Choeurng V, Howard L, De Hoedt A, du Plessis M, Yousefi K, Lam LL, Buerki C, Ra S, Robbins B, Trabulsi EJ, Shah NL, Abdollah F, et al. Utilization of a genomic classifier for prediction of metastasis following salvage radiation therapy after radical prostatectomy. Eur Urol. 2016; 70:588-96. doi:10.1016/j.eururo.2016.

30. Glass AG, Leo MC, Haddad Z, Yousefi K, du Plessis M, Chen C, Choeurng V, Abdollah F, Robbins B, Ra S, RichertBoe KE, Buerki C, Pearson K, et al. Validation of a genomic classifier for predicting post-prostatectomy recurrence in a community based health care setting. J Urol. 2015; 195:1748-53. doi:10.1016/j.juro.2015.11.044.

31. Den RB, Yousefi K, Trabulsi EJ, Abdollah F, Choeurng V, Feng FY, Dicker AP, Lallas CD, Gomella LG, Davicioni E, Karnes RJ. Genomic classifier identifies men with adverse pathology after radical prostatectomy who benefit from adjuvant radiation therapy. J Clin Oncol. 2015; 33:944-51. doi:10.1200/JCO.2014.59.0026.

32. Klein EA, Cooperberg MR, Magi-Galluzzi C, Simko JP, Falzarano SM, Maddala T, Chan JM, Li J, Cowan JE, Tsiatis AC, Cherbavaz DB, Pelham RJ, Tenggara-Hunter I. A 17-gene assay to predict prostate cancer aggressiveness in the context of Gleason grade heterogeneity, tumor multifocality, and biopsy undersampling. Eur Urol. 2014; $66: 550-60$. 